\title{
Propagation of monochromatic light in a hot and dense medium
}

\author{
Samina S. Masood ${ }^{\mathrm{a}}$ \\ Department of Physical and Applied Sciences, University of Houston Clear Lake, Houston, TX 77058, USA
}

Received: 24 October 2017 / Accepted: 20 November 2017 / Published online: 4 December 2017

(c) The Author(s) 2017. This article is an open access publication

\begin{abstract}
Photons, as quanta of electromagnetic fields, determine the electromagnetic properties of an extremely hot and dense medium. Considering the properties of the photons in the interacting medium of charged particles, we explicitly calculate the electromagnetic properties such as the electric permittivity, magnetic permeability, refractive index and the propagation speed of electromagnetic signals in an extremely hot and dense background. Photons acquire a dynamically generated mass in such a medium. The screening mass of the photon, the Debye shielding length and the plasma frequency are calculated as functions of the statistical parameters of the medium. We study the properties of the propagating particles in astrophysical systems of distinct statistical conditions. The modifications in the properties of the medium lead to the equation of state of the system. We mainly calculate all these parameters for extremely high temperatures of the early universe.
\end{abstract}

\section{Introduction}

We re-investigate the behavior of the first generation of leptons and the corresponding electromagnetic field in extremely hot and dense medium of the electrons below the electroweak temperature. This system is composed of different types of particles but the significant contribution comes from the light particles with masses lower than the existing temperature in the early universe. The overall behavior of particles in a medium is a net result of interaction of propagating particles with the medium. The background corrections become much more significant at high temperatures and densities where the propagating particles can modify the properties of the medium [1-6]. We consider the light mass particles in a heat bath of the electrons and photons at temperature below the decoupling temperature. Thus the higher generations of leptons are not expected to be affected signif-

\footnotetext{
a e-mail: masood@uhcl.edu
}

icantly by temperatures that are much below their masses, or to affect these themselves. Radiative background corrections due to the heavy intermediate vector bosons of electroweak interactions are also suppressed because of their heavy mass. Therefore, we study the background contribution of radiation while it is interacting with matter and the temperature of hot electrons is below $2 \mathrm{MeV}$, the decoupling temperature. The system is considered to be in thermal equilibrium in specified regions of the stellar bodies.

In this paper, we study a pure gas of the electrons and photons that can be converted into electromagnetic plasma of the photons and electrons at high temperatures which are sufficiently smaller than the $\mathrm{W}$ and $\mathrm{Z}$ masses. Background contributions of interactions between electrons and photons in the medium are incorporated through statistically corrected propagators with the distribution function of the photons and electrons in the medium. However, electromagnetic properties of the propagating neutrinos (with nonzero mass) will be modified due to the electron's induced magnetic moment. The heat bath with a high concentration of the electrons and photons can still be considered as a relativistic plasma of the electrons and photons (for $\mu \ll m$ ). Photon acquires a temperature dependent screening mass and a Debye screening length which can be calculated from the longitudinal component of the vacuum polarization tensor. The electromagnetic properties of the medium are modified which may cause the phase transition under suitable conditions. The photon can then be treated as a plasmon as they transfer certain amount of energy to electrons and are responsible for the radiative corrections. The plasmon at higher energy can decay into a neutrino-antineutrino pair, which can couple with the photons in a medium through electrons as a higher order effect. The magnetic moment is a perturbative effect. First order radiative corrections in Fig. 1 indicate the magnetic moment of the electron (Fig. 1a) and neutrino (Fig. 1b, c). Electron induces a nonzero magnetic moment to neutrino due to the interaction of the electron with the neutrino in $\mathrm{e}^{-} v_{e} \rightarrow \mathrm{e}^{-} v_{e}$ in the minimal standard models with very tiny mass of the 


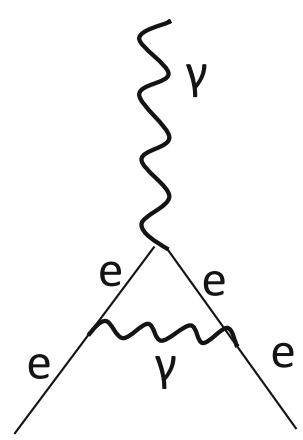

(a)

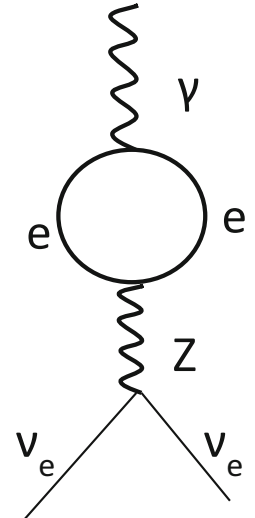

(b)

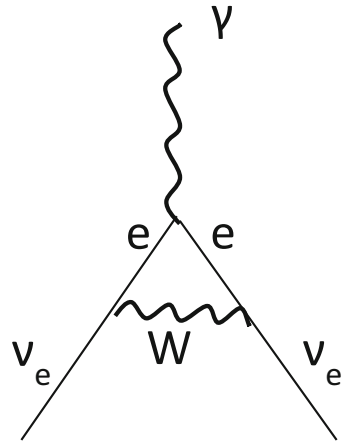

(c)
Fig. 1 First order radiative corrections to plasmons in the electroweak model results into a coupling of the photon with electron or neutrino or the interaction of leptons with the magnetic field. Radiative corrections to electromagnetic vertex (a), the tadpole diagram (b) corresponds to a neutral current and gives a nonzero contribution for an asymmetric combination of an electron-positron background only. The bubble diagram (c) gives a major contribution to the magnetic dipole moment of the neutrino neutrino, as the magnetic moment is the property of matter. Thermal background corrections due to the second or higher generation of particles will always be suppressed even if those particles are injected in the medium from outside.

\section{Calculational scheme}

The properties of the electrons as electromagnetically interacting particles in an extremely hot and dense system are studied using the renormalization scheme of quantum electrodynamics (QED) in statistical media for different ranges of temperature and chemical potential [7-13]. We use the renormalization scheme of QED in a real-time formalism. This scheme is valid in a heat bath of real particles below the decoupling temperature. All of the Feynman rules of QED remain unchanged. The statistical effects are included through the statistical distribution functions. Massless vector boson interaction is incorporated by the Bose-Einstein distribution function and the Fermi-Dirac distribution function [14] is used for the fermionic contributions,

$n_{F}(p, \mu)=\left[\frac{\theta\left(p_{0}-\mu\right)}{e^{\beta\left(p_{0}-\mu\right)}+1}+\frac{\theta\left(p_{0}+\mu\right)}{e^{\beta\left(p_{0}+\mu\right)}+1}\right]$.

Equation (1) corresponds to a closed system with equal and opposite chemical potential $(\mu)$ of the fermions and antifermions in a CP symmetric background. The first term in parenthesis corresponds to the particle distribution, whereas the second term corresponds to the antiparticle distribution in hot and dense medium. It is convenient to expand the distribution functions of particle and antiparticle in powers of $m \beta$ (for constant $\mu$ ), where $m$ is the mass of the corresponding particles and $\beta=1 / T$. All the statistical parameters $\mu, T$ and $\beta$ are expressed in units of $m$, the electron mass. In a heat bath of the electrons, at very high temperatures, the properties of the electrons are modified corresponding to temperature and density of the system. The physically measurable values of the electron mass, charge and wave function of the electrons in a medium are calculated as renormalization constants [6] of QED in a hot and dense heat bath for different ranges of temperature and chemical potential. Without getting into details of the calculations, we use the physically measurable parameters of the propagating particle with the renormalization constants of QED to determine the electromagnetic properties of the medium which can be converted into a relativistic plasma. The thermal contributions (calculated in a real-time formalism) to the renormalization constants of QED are combined with the relevant renormalization constants in vacuum to find the renormalized parameters of the theory in a statistical medium. These renormalized finite quantities correspond to the physically measurable values of the parameters such as electron mass [7,8], charge [9-11], wave function [12] and the magnetic moments [13,15-21]. We can then replace the rest mass of the electron by the physically measurable renormalized mass:

$m^{R}=m^{0}\left(1+\frac{\delta m}{m}\right)$

$m^{R}=m^{\text {Phys }}$

The superscripts $R$ and Phys correspond to the renormalized parameters and physically measurable quantities, respectively. The corresponding relations between renormalized wave function of electron and that of the corresponding vacuum value are given as

$\psi^{R}=\frac{1}{\sqrt{Z_{2}}} \psi^{0}$

$\psi^{R}=\psi^{\text {Phys }}$ 
such that the probability of finding particles in certain states becomes a function of the statistical parameters of the medium. The electromagnetic fields is expressed as

$$
\begin{aligned}
& \left(A^{R}\right)_{\mu}=\frac{1}{\sqrt{Z_{3}}}\left(A^{0}\right)_{\mu} \\
& \left(A^{R}\right)_{\mu}=\left(A^{\text {Phys }}\right)_{\mu}
\end{aligned}
$$

and the physical mass Eq. (2a), wave function Eq. (3a) and the electromagnetic fields Eq. (4a) give the physically measurable values of the corresponding parameters in a hot and dense medium. The QED Lagrangian of such a system can then be written as

$$
\begin{aligned}
L= & -\frac{1}{4} Z_{3} F_{\alpha \mu \nu} F_{\alpha}^{\mu \nu}+i Z_{2} \bar{\psi}_{R} \gamma_{\mu} D^{\mu} \psi_{R}+Z_{2} \overline{\psi_{R}} m_{0} \psi_{R} \\
& +e_{0} Z_{2} \sqrt{Z_{3}} \overline{\psi_{R}} \gamma_{\mu} A_{R}^{\mu} \psi_{R} .
\end{aligned}
$$

In this scheme of calculations, the renormalization constants of QED are considered to be the effective parameters of the theory. The renormalization constants of QED give the physical mass and the charge of the electrons and the corresponding wave function at finite temperature and density. The vacuum polarization tensor $\Pi_{\mu \nu}$ for such a system can be written by replacing the photon and electron propagator in vacuum by the one in the medium in a real-time formalism such that

$$
\begin{aligned}
& \Pi_{\mu \nu}(K, \mu)=i e^{2} \int \frac{\mathrm{d}^{4} p}{(4 \pi)^{4}} \operatorname{Tr}\left\{\gamma_{\mu}(p+K+m) \gamma_{\nu}(p+\mathrm{m})\right\} \\
& \quad \times\left[\frac{1}{(p+K)^{2}-m^{2}}+\Gamma_{F}(p+K, \mu)\right] \\
& \quad \times\left[\frac{1}{p^{2}-m^{2}}+\Gamma_{F}(p, \mu)\right]
\end{aligned}
$$

with [14]

$$
\begin{aligned}
\Gamma_{F}(p, \mu)= & 2 \pi i \delta\left(p^{2}-m^{2}\right)\left[\theta\left(p_{0}\right) n_{F}(p, \mu)\right. \\
& \left.+\theta\left(-p_{0}\right) n_{F}(p,-\mu)\right],
\end{aligned}
$$

whereas $K_{\alpha}$, the 4-momentum of the photon, is related to the energy $\omega$ and momentum $k$ :

$K^{2}=\omega^{2}-k^{2}, \quad \omega=K_{\alpha} u^{\alpha}$.

$K^{2}=0$ is in vacuum due to the transverse nature of light which ensures the absence of longitudinal component as well as the masslessness of the photon. $u^{\alpha}(=(1,0,0,0))$ Correspond to the 4-velocity of the heat bath.

The vacuum part $\Pi_{\mu \nu}^{T=0}(K)$ and the medium contribution $\Pi_{\mu \nu}^{\beta}(K, \mu)$ to Eq. (6) can be written as

$$
\Pi_{\mu \nu}(K)=\Pi_{\mu \nu}^{T=0}(K)+\Pi_{\mu \nu}^{\beta}(K, \mu)
$$

with

$$
\begin{aligned}
& \Pi_{\mu \nu}^{\beta}(K, \mu)=-\frac{2 \pi e^{2}}{2} \int \frac{\mathrm{d}^{4} p}{(4 \pi)^{4}} \operatorname{Tr}\left\{\gamma_{\mu}(p+K+m) \gamma_{\mu}(p+m)\right\} \\
& \times\left[\frac{\delta\left[(p+K)^{2}-m^{2}\right]}{p^{2}-m^{2}}\left\{n_{F}(p+K, \mu)+n_{F}(p+K,-\mu)\right\}\right. \\
& \left.+\frac{\delta\left[p^{2}-m^{2}\right]}{(p+K)^{2}-m^{2}}\left\{n_{F}(p, \mu)+n_{F}(p,-\mu)\right\}\right] .
\end{aligned}
$$

The polarization tensor $\Pi_{\mu \nu}^{\beta}(K, \mu)$ can generally be written in terms of the longitudinal and transverse components $\Pi_{\mathrm{L}}(k, \omega)$ and $\Pi_{\mathrm{T}}(k, \omega)$, respectively, such that it satisfies the relation

$\Pi_{\mu \nu}(K, \mu)=P_{\mu \nu} \Pi_{\mathrm{T}}(K, \mu)+Q_{\mu \nu} \Pi_{\mathrm{L}}(K, \mu)$,

Whereas the transverse component of the polarization tensor $P_{\mu \nu}$ can be written in terms of the parameters of the statistical medium:

$P_{\mu \nu}=\tilde{g}_{\mu \nu}+\frac{\tilde{K}_{\mu} \tilde{K}_{v}}{k^{2}}$

and the longitudinal component of the polarization tensor $Q_{\mu \nu}$ is expressed in the statistical medium:

$Q_{\mu \nu}=-\frac{1}{K^{2} k^{2}}\left(k^{2} u_{\mu}+\omega \tilde{K}_{\mu}\right)\left(k^{2} u_{\nu}+\omega \tilde{K}_{\nu}\right)$

whereas,

$\tilde{g}_{\mu \nu}=g_{\mu \nu}-u_{\mu} u_{\nu}$

and

$\tilde{K}_{\mu}=K_{\mu}-\omega u_{\mu}$

Such that they satisfy the conditions:

$P_{\nu}^{\mu} P_{\alpha}^{\mu}=P_{\alpha}^{\mu} \quad Q_{\alpha}^{\mu}=Q_{v}^{\mu} Q_{\alpha}^{v} \quad K_{\mu} P_{v}^{\mu}=0 \quad K_{\mu} Q_{v}^{\mu}=0$

The structure of the vacuum polarization tensor becomes clearer if we put it in the component form,

$P_{\mu \nu}=-\left(\begin{array}{cc}0 & P_{0 j} \\ P_{i 0} & P_{i j}\end{array}\right)$.

Defining $b_{i j}=\frac{k_{i} k_{j}}{K^{2}}$, the elements of the polarization tensor $P_{\mu \nu}$ can be expressed as

$P_{i 0}=P_{0 j}=0$ and $P_{i j}=\delta_{i j+} b_{i j}$,

whereas the elements of the polarization tensor $Q_{\mu \nu}$ attain the form

$Q_{\mu \nu}=-\left(\begin{array}{cc}\frac{-k^{2}}{K^{2}} & Q_{0 j} \\ Q_{i 0} & Q_{j j}\end{array}\right)$

and

$Q_{0 j}=-i \omega b_{j} \quad Q_{i 0}=-i \omega b_{i} \quad Q_{i j}=\frac{\omega^{2}}{K^{2}}$ 
with

$\Pi_{\mathrm{L}}(\omega, k)=0$

in vacuum. In the absence of the longitudinal component of the photon (in vacuum), Eq. (11) reduces to

$\Pi_{\mu \nu}=P_{\mu \nu}$,

showing that all the light is transversally polarized such that $\varepsilon(K)=1$ and $\mu(K)=1$, as well. Transversality of photon is the property associated with the masslessness of the photon. When the photon acquires a plasma screening mass at nonzero temperature, it gives a nonzero contribution to the longitudinal component of the polarization tensor, which results in slow down or trapping of the electromagnetic signal in a medium. The reduction in the speed of electromagnetic waves depends on the statistical properties of the medium. A nonzero parallel component in three dimensional space can still maintain the circular polarization in an isotropic medium and the possibility of trapping of light in a medium affects the transverse propagation in an anisotropic medium in extreme statistical conditions. Due to the mass of the photon light slows down in the transverse direction by losing some energy to longitudinal direction.

Temperature and density corrections to the QED parameters in a hot and dense medium are reviewed in the next section. The magnetic field is not included explicitly. However, in the presence of charged leptons at high temperatures, significantly large magnetic field is expected. In a closed system with isotropic matter distribution, a constant magnetic field is expected at a constant temperature. Therefore, at a given temperature, magnetic field effect is incorporated through the potential energy contribution to the charged leptons and the energy of particles will be modified in the presence of the magnetic field as

$$
E^{2}=p_{0}^{2}=p^{2}+m^{2} \pm \mu N+(2 \ell+1) e B
$$

where $\ell$ corresponds to the Landau level and $B$ is a constant magnetic field. $B$ can be replaced by the time varying magnetic field to incorporate the change in magnetic energy with time. We postpone a detailed study of this effect of different type of magnetic fields for now.

\section{QED parameters in a medium}

The thermal corrections to the QED parameters can be written as a function of temperature $\mathrm{T}$ and chemical potential $\mu$ in the form of Masood's $a, b, c, \ldots$ functions expressed as $a_{i}(m \beta, \mu)$ [6-23] and referred to as Masood's functions hereafter,

$$
\begin{aligned}
\frac{\delta m}{m} \approx & \frac{\alpha \pi T^{2}}{3 m^{2}}\left[1-\frac{6}{\pi^{2}} c(m \beta, \mu)\right]+\frac{2 \alpha}{\pi} \frac{T}{m} a(m \beta, \mu) \\
& -\frac{3 \alpha}{\pi} b(m \beta, \mu) .
\end{aligned}
$$

The first term in brackets is a measure of thermal corrections related to the increase in kinetic energy due to the coupling of particles with radiation, whereas the $a, b$ and $c$ functions are evolved from the integration of the Fermi-Dirac distribution, and it vanishes at low temperature where the presence of hot fermions is negligible in the system. We have

$$
\begin{aligned}
& a(m \beta, \pm \mu)=\ln \left(1+e^{-\beta(m \pm \mu)}\right), \\
& b(m \beta, \pm \mu)=\sum_{n=1}^{\infty}(-1)^{n} e^{\mp \beta \mu} E i(-n m \beta), \\
& c(m \beta, \pm \mu)=\sum_{n=1}^{\infty}(-1)^{n} \frac{e^{-n \beta(m \pm \mu)}}{n^{2}},
\end{aligned}
$$

where $+(-) \mu$ correspond to the chemical potential of the fermion (antifermion) in the medium. The wave function renormalization constant of QED can be written as [6]

$$
\begin{aligned}
Z_{2}^{-1}(\beta)= & Z_{2}^{-1}(T=0)-\frac{2 \alpha}{\pi} \int_{0}^{\infty} \frac{\mathrm{d} k}{k} n_{B}(k)-\frac{5 \alpha}{\pi} b(m \beta, \mu) \\
& +\frac{\alpha T^{2}}{\pi v E^{2}} \ln \frac{1+v}{1-v}\left\{\frac{\pi^{2}}{6}+m \beta a(\mathrm{~m} \beta, \mu)-c(\mathrm{~m} \beta, \mu)\right\},
\end{aligned}
$$

and the charge renormalization constant is calculated [6] to be

$$
\begin{aligned}
Z_{3} \cong & 1+\frac{2 e^{2}}{\pi^{2}}\left[\frac{m a(m \beta, \mu)}{\beta}-\frac{c(m \beta, \mu)}{\beta^{2}}\right. \\
& \left.+\frac{1}{4}\left(m^{2}+\frac{1}{3} \omega^{2}\right) b(m \beta, \mu)\right] .
\end{aligned}
$$

The photon in the medium develops a plasma screening mass which can be obtained from the longitudinal and transverse component of the vacuum polarization tensor $\Pi_{\mathrm{L}}(0, k)$ and $\Pi_{\mathrm{T}}(k, k)$ where $K^{2}=\omega^{2}-k^{2}$ and corresponds to monochromatic light.

In this scheme of calculations, longitudinal and transverse components $\left(\Pi_{\mathrm{L}}\right.$ and $\Pi_{\mathrm{T}}$, respectively) of the vacuum polarization tensor $\Pi_{\mu \nu}$ play a crucial role in the calculation of the electromagnetic properties of a medium. The electromagnetic properties such as electric permittivity $\varepsilon(K)$ and magnetic permeability $\mu(K)$ as well as the refractive index, propagation speed and the magnetic moment of different particles in the medium are studied by using these basic properties of the medium. The electric permittivity $\varepsilon(K)$ and the magnetic permeability $\mu(K)$ are related [9] to $\Pi_{\mathrm{L}}$ and $\Pi_{\mathrm{T}}$ : 
$\varepsilon(K)=1-\frac{\Pi_{\mathrm{L}}}{K^{2}}$,

$\frac{1}{\mu(K)}=1+\frac{K^{2} \Pi_{\mathrm{T}}-\omega^{2} \Pi_{\mathrm{L}}}{k^{2} K^{2}}$,

such that

$$
\begin{aligned}
\Pi_{\mathrm{L}} \cong & \frac{4 e^{2}}{\pi^{2}}\left(1-\frac{\omega^{2}}{\mathbf{k}^{2}}\right)\left[\left(1-\frac{\omega}{2 \mathbf{k}} \ln \frac{\omega+\mathbf{k}}{\omega-\mathbf{k}}\right)\right. \\
& \times\left(\frac{m a(m \beta, \mu)}{\beta}-\frac{c(m \beta, \mu)}{\beta^{2}}\right) \\
& \left.+\frac{1}{4}\left(2 m^{2}-\omega^{2}+\frac{11 \mathbf{k}^{2}+37 \omega^{2}}{72}\right) b(m \beta, \mu)\right]
\end{aligned}
$$

$$
\begin{aligned}
\Pi_{\mathrm{T}} \cong & \frac{2 e^{2}}{\pi^{2}}\left[\left\{\frac{\omega^{2}}{\mathbf{k}^{2}}+\left(1-\frac{\omega^{2}}{\mathbf{k}^{2}}\right) \frac{\omega}{2 \mathbf{k}} \ln \frac{\omega+\mathbf{k}}{\omega-\mathbf{k}}\right\}\right. \\
& \times\left(\frac{m a(m \beta, \mu)}{\beta}-\frac{c(m \beta, \mu)}{\beta^{2}}\right) \\
& \left.+\frac{1}{8}\left(2 m^{2}+\omega^{2}+\frac{107 \omega^{2}+131 \mathbf{k}^{2}}{72}\right) b(m \beta, \mu)\right] .
\end{aligned}
$$

Whereas at extremely high temperatures, $\omega=k$ is not allowed because the above equations are valid at high temperature only. At those temperatures, longitudinal component cannot vanish so $\ln (0)$ singularity is ruled out and the Debye shielding length turns out to be

$$
\begin{aligned}
& \prod_{\mathrm{L}}(0, k) \equiv K_{\mathrm{L}}^{2} \cong \frac{e^{2} T^{2}}{3} \\
& \lim _{k \rightarrow 0} \\
& \prod_{\mathrm{T}}(k, \vec{k}) \equiv \omega_{\mathrm{T}}^{2} \cong \frac{e^{2} T^{2}}{6}(T \gg m),
\end{aligned}
$$

whereas, in the relativistic plasma, it depends on the temperature quadratically. Now the electric permittivity $\varepsilon(K)$ and magnetic permeability $\mu(K)$ of such a medium can be calculated from the longitudinal and transverse components [9] to be

$$
\begin{aligned}
\varepsilon(K) \cong & 1-\frac{4 e^{2}}{\pi^{2} K^{2}}\left(1-\frac{\omega^{2}}{\mathbf{k}^{2}}\right)\left\{\left(1-\frac{\omega}{2 \mathbf{k}} \ln \frac{\omega+\mathbf{k}}{\omega-\mathbf{k}}\right)\right. \\
& \times\left(\frac{m a(m \beta, \mu)}{\beta}-\frac{c(m \beta, \mu)}{\beta^{2}}\right) \\
& \left.+\frac{1}{4}\left(2 m^{2}-\omega^{2}+\frac{11 k^{2}+37 \omega^{2}}{72}\right) b(m \beta, \mu)\right\}
\end{aligned}
$$

and

$$
\begin{aligned}
\frac{1}{\mu(K)} \cong & 1-\frac{2 e^{2}}{\pi^{2} k^{2} K^{2}}\left[\omega ^ { 2 } \left\{\left(1-\frac{\omega^{2}}{\mathbf{k}^{4}}-\left(1+\frac{\mathbf{k}^{2}}{\omega^{2}}\right)\right.\right.\right. \\
& \left.\times\left(1-\frac{\omega^{2}}{\mathbf{k}^{2}}\right) \frac{\omega}{2 \mathbf{k}} \ln \frac{\omega+\mathbf{k}}{\omega-\mathbf{k}}\right\} \\
& \times\left(\frac{m a(m \beta, \mu)}{\beta}-\frac{c(m \beta, \mu)}{\beta^{2}}\right) \\
& \left.\left.-\frac{1}{8}\left(6 m^{2}-\omega^{2}+\frac{129 \omega^{2}-109 k^{2}}{72}\right) b(m \beta, \mu)\right\}\right]
\end{aligned}
$$

Equations (21) and (23) show the dependence of the longitudinal and transverse components of the polarization as well as the electric permittivity and the magnetic permeability of the medium as a function of $\omega$ and $k$ corresponding to the propagating electromagnetic waves.

The dependence of $\varepsilon(K)$ and $\mu(K)$ on the temperature induces temperature dependence to the propagation velocity and the refractive index of the medium. These quantities also vary with the energy and the momentum of the photons. This is a distinct feature of the extremely hot medium of the early universe that the refractive index will depend on the wave properties as well as the temperature of the medium. The speed of propagation of electromagnetic waves in such a medium can be expressed as

$v_{\text {prop }}=\sqrt{\frac{1}{\varepsilon(K) \mu(K)}}$

and the refractive index of the medium turns out to be

$n=\frac{c}{v}=\sqrt{\frac{\varepsilon(K) \mu(K)}{\varepsilon_{0}(K) \mu_{0}(K)},}$

$\lambda_{\mathrm{D}}=\Pi_{\mathrm{L}}$

It is well known that a nonzero longitudinal component in the early universe corresponds to the Debye length and should be a quadratic function of temperature whereas in a classical plasma the temperature dependence of the shielding length reads

$\lambda_{\mathrm{D}}=\left(\frac{\varepsilon k_{\mathrm{B}} T}{\sum_{j=1}^{N} n_{j}^{0} q_{j}^{2}}\right)^{1 / 2}$.

\section{Propagation of light in the early universe}

It is well known from the thermal history of the universe that temperature of the universe was extremely high and the chemical potential was extremely low in this radiation dominated era. Therefore, the valid limit of temperature in this situation is $T \gg m \gg \mu$. In this section, we evaluate all 
Masood's functions for extremely high temperatures in the given ranges of the photon momenta and energy. The dominant thermal contribution comes from the interaction with the radiation at the equilibrium temperature $T$ and fermions at the same temperature. The values of $a_{i}(m \beta, \mu)$ for extremely high temperature, only the $c(m \beta, \mu)$ term contributes, giving

$c(m \beta, \mu)=-\frac{\pi^{2}}{12}$.

And in the extremely high temperature limit $(T \gg m \gg$ $\mu$ ) with ignorable density the longitudinal and transverse components of the vacuum polarization tensor can be written as

$$
\begin{aligned}
& \varepsilon(K) \cong 1-\left(1-\frac{\omega^{2}}{\mathbf{k}^{2}}\right)\left(1-\frac{\omega}{2 \mathbf{k}} \ln \frac{\omega+\mathbf{k}}{\omega-\mathbf{k}}\right) \frac{4 \pi \alpha T^{2}}{3 K^{2}}, \\
& \frac{1}{\mu(K)} \cong 1+\frac{2 \pi \alpha T^{2}}{3 K^{2}} \frac{\omega^{2}}{k^{2}}\left\{\left(1-\frac{\omega^{4}}{\mathbf{k}^{4}}-\left(1+\frac{\mathbf{k}^{2}}{\omega^{2}}\right)\right.\right. \\
&\left.\times\left(1-\frac{\omega^{2}}{\mathbf{k}^{2}}\right) \frac{\omega}{2 \mathbf{k}} \ln \frac{\omega+\mathbf{k}}{\omega-\mathbf{k}}\right\} .
\end{aligned}
$$

Equation (27) can be evaluated, using (26), for two extreme conditions based on the properties of light:

(i) For large energy of the photons, $\omega \gg k$

$$
\begin{aligned}
& \pi_{\mathrm{L}}=-\frac{\omega^{2} e^{2} T^{2}}{3 k^{2}} \pi_{\mathrm{T}}=\frac{\omega^{2} e^{2} T^{2}}{6 k^{2}} \\
& \varepsilon(k)=1+\frac{\omega^{2} e^{2} T^{2}}{3 k^{4}} \frac{1}{\mu(k)} \cong \frac{\omega^{4} e^{2} T^{2}}{3 k^{4} K^{2}}, \\
& v_{\text {prop }}=\sqrt{\frac{\omega^{4} e^{2} T^{2}}{K^{2}\left(3 k^{4}+\omega^{2} e^{2} T^{2}\right)}},
\end{aligned}
$$

and the refractive index of the medium turns out to be

$$
n=\frac{c}{v}=\sqrt{\frac{K^{2}\left(3 k^{4}+\omega^{2} e^{2} T^{2}\right)}{\omega^{4} e^{2} T^{2}}} .
$$

(ii) For large momentum of the photons, $\omega \ll k$

$$
\begin{aligned}
& \pi_{\mathrm{L}}=\frac{e^{2} T^{2}}{3} \pi_{\mathrm{T}}=\frac{e^{2} T^{2}}{6}, \\
& \varepsilon(k)=1-\frac{e^{2} T^{2}}{6 k^{2}} \quad \frac{1}{\mu(k)} \cong \frac{e^{2} T^{2}}{6 K^{2}} .
\end{aligned}
$$

The thermal correction to the speed of the propagation of light in such a medium is

$$
v_{\text {prop }}=\sqrt{\frac{e^{2} T^{2}}{\left(6 k^{2}-e^{2} T^{2}\right)}} .
$$

The refractive index of this medium is given by

$n=\sqrt{\frac{\left(6 k^{2}-e^{2} T^{2}\right)}{e^{2} T^{2}}}$.

Equation (34) puts a natural limit on the values of $k$. Moreover, $k^{2}=\frac{e^{2} T^{2}}{6}$ is not physically allowed as it will give an infinite speed and zero refractive index. These equations cannot be true for $T=0$ as well. The values of the electric permittivity and magnetic permeability depend on the values of the plasmon frequency and the wave number at a given temperature. The propagation speed $v_{\text {prop }}$ and the refractive index $n$ become a function of temperature, energy and momentum of the photon. It is also to be noticed that the thermal contributions in Eq. (27)) start at $T>0.5 \mathrm{MeV}$. The electromagnetic properties of the medium cannot see any thermal contributions after the nucleosynthesis $[13,15]$ is over in the early universe. This limit is below the neutrino decoupling temperature, indicating that the nucleosynthesis started right after the temperature of the universe dropped below the neutrino decoupling temperature and the neutrino capture contributed to beta processes during nucleosynthesis. All the calculations in this paper are relevant for the temperatures below the neutrino decoupling temperatures and higher than the electron mass. In other words, these calculations are valid after the neutrino decoupling and before the nucleosynthesis totally stops. It shows that the properties of the medium change tremendously during nucleosynthesis.

The existence of hot electrons in a medium at such temperatures ensures a significant effect on physically measurable values of the electron mass, charge and concentration of the electrons in a medium, which leads to the change in the electromagnetic properties in terms of magnetic moments of leptons, electric permittivity and magnetic permeability of the medium. The electromagnetic properties of a medium then work differently on the particles that propagate through this medium.

\section{The magnetic moment of charged particles}

Relativistically moving charged particles have an associated electric field and their relativistic motion at high temperatures create weak non-negligible currents in extreme situations in a medium. When charged particles are accelerated in a medium and a continuous change in energy occurs through acceleration of particles along with the change in temperature of the system, an associated magnetic field is generated. In such an electromagnetic system, there are localized electromagnetic fields that are associated with the distribution of charged particles in the medium. The magnetic moment is associated with charge and mass of leptons. Lighter particles have large magnetic moment effect and thermal contribution is higher 
for the lighter particles as temperature is always compared to the mass of the particles for thermal effects. Renormalization scheme gives a change in mass in Eq. (2). The charge of the electron is not affected until the temperature is extremely high as indicated in Eq. (5).

The magnetic moment is simply calculated from the change in mass in thermal background given as

$$
\begin{aligned}
a_{\mu} & =\left(\frac{\alpha}{2 \pi}-\frac{2}{3} \frac{\delta m}{m}\right) \mu_{B} \\
\frac{\delta m}{m} & =\frac{\delta m}{m}(\text { vacuum })+\frac{\delta m}{m}(T, \mu)
\end{aligned}
$$

$\mu_{\mathrm{B}}$ is the unit of magnetic moment called Bohr Magneton. So the statistical corrections to the magnetic moment of the electron is directly proportional to the statistical self-mass corrections, given in Eq. (16).

Generalization of results A straightforward generalization of all the above results can be done by evaluating Masood's functions $a_{i}$ such that $a_{1}=a(m \beta, \mu), a_{2}=b(m \beta, \mu), a_{3}=$ $c(m \beta, \mu)$, and so on. All of the Masood's statistical functions $a_{i}$ correspond to electron background contributions in the above equations and always correspond to fermion background contribution for $T>\mu$. Evaluating the above equations, we just consider the electron background as $m$ is taken as the electron mass. Thus these functions correspond to the electron background for temperatures higher than the electron mass. The positrons' contribution in the same medium can be expressed by replacing $\mu$ with a negative $\mu$. Everything else remains unchanged. Net contributions from the $\mathrm{CP}$ symmetric background can be obtained by taking the average of particle and antiparticle background contributions and the net background contributions can be obtained by replacing the $a_{i}$ functions by the corresponding difference functions, giving [6]

$$
\begin{aligned}
& a_{\mathrm{avg}}(m \beta, \mu)=\frac{1}{2}[a(m \beta, \mu)+a(m \beta,-\mu)], \\
& a_{\mathrm{net}}(m \beta, \mu)=\frac{1}{2}[a(m \beta, \mu)-a(m \beta,-\mu)],
\end{aligned}
$$

such that, in the limit $T \gg \mu$, the chemical potential contribution is ignorable and hence the thermal effects dominate, such that

$$
\begin{aligned}
& a_{\text {net }}\left(m_{\ell} \beta, \mu\right) \rightarrow 0, \\
& a_{\text {avg }}\left(m_{\ell} \beta, \mu\right) \rightarrow a_{i}\left(m_{\ell} \beta\right) .
\end{aligned}
$$

The average background contribution can be obtained by the corresponding functions considering Eq. (17),

$$
\begin{aligned}
& a_{\mathrm{avg}}\left(m_{\ell} \beta, \mu\right) \cong \frac{1}{2} \ln \left[\left(1+e^{-\beta\left(m_{\ell}-\mu\right)}\right)\left(1+e^{-\beta\left(m_{\ell}+\mu\right)}\right)\right], \\
& b_{\mathrm{avg}}\left(m_{\ell} \beta, \mu\right) \cong \sum_{n=1}^{\infty}(-1)^{n} \cosh (n \beta \mu) \operatorname{Ei}\left(-n m_{\ell} \beta\right), \\
& c_{\mathrm{avg}}\left(m_{\ell} \beta, \mu\right) \cong \sum_{n=1}^{\infty}(-1)^{n} \cosh (n \beta \mu) \frac{e^{-n \beta m_{\ell}}}{n^{2}} .
\end{aligned}
$$

It can easily be seen that at extremely high temperatures, the fermion contribution from the medium is controlled by $c(m \beta, \mu)$ and is given in Eq. (26).

\section{Results and discussions}

We reiterate some results from the previously calculated renormalization constants of QED in extremely hot and dense media using a real-time formalism. Equations (16)-(19) give hot and dense background corrections to the electron mass, wave function and charge comes, making renormalization constants as a part of physically measurable parameters of the statistical system. It can easily be seen that thermal behavior at high temperatures is clearly a quadratic behavior and all the parameters of the system becomes functions of temperature with $(T / m)^{2}$, as dominant contribution. The photon background contribution is always proportional to $(T / m)^{2}$ whereas the dominant fermion contribution is also the same way at very high temperatures. It has already been noticed that all the parameters behave in a more complicated way during the nucleosynthesis $[13,15]$ in the early universe and depend on Masood's functions. The thermal contribution to the electron mass, wave function and charge of the electron at high temperature is plotted in Fig. 2, showing a comparison between all three renormalization parameters of QED. The mass dependence is substantial and is non-ignorable even at $T<m$. However, charge renormalization is not contributed if enough fermions are not available in the system for the photon interaction, so a background effect cannot be seen below the temperatures of $10^{10} \mathrm{~K}$, i.e., equal to the electron mass. All the parameters have a dominant quadratic dependence on the temperature with different coefficients. The thermal contribution to the electron self-mass is a couple of orders of magnitude greater than the electron charge and the coupling constant of QED. It can be seen from Eqs. (16)-(19) that the wave function correction is much smaller than the electron mass or even the charge of the electron. It can easily be seen that the mass contribution dominates over the charge contribution because electron mass has radiative corrections by its interaction with the radiation (photon) in the background. However, charge does not see the photon and thermal contribution is only due to the fermion background. Thermal corrections to the electron wave function are very 

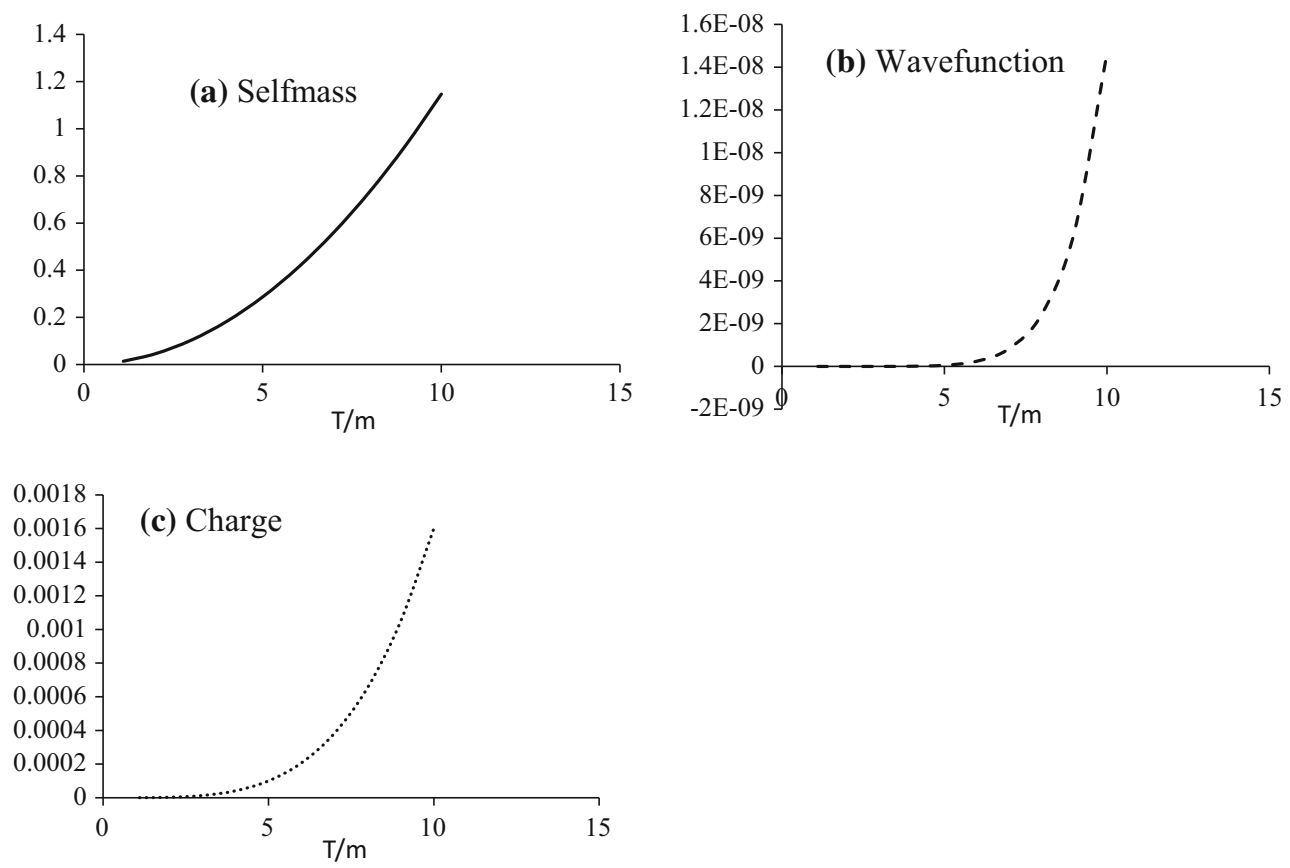

Fig. 2 The properties of the electrons as a function of temperature (in units of $\mathrm{m}$ ). The thermal corrections to a electron mass; $\mathbf{b}$ wave function; and charge

small even at $T>m$. However, the charge of the electron is not affected by the background at low temperatures $(T<m)$ because massless photons do not interact among themselves. At $T>m$, the photon acquires a dynamically generated mass which increases with temperature in the presence of a high concentration of electrons in a medium. The QED parameters indicating properties of the electrons at high temperature are plotted in Fig. 2.

Self-mass corrections to electron affect the magnetic moment of electron which is also proportional to the $T^{2} / \mathrm{m}^{2}$. Figure 3 gives the magnetic moment of the electron as a function of temperature as the electron mass keeps on increasing with temperature. However, the presence of charged fermions in the background affects the electromagnetic properties of the electron also. Figure 3 plots the thermal contributions to the magnetic moment as compared to the thermal contribution to the mass of electron.

The magnetic moment is a form factor and is a property of the mass and charge whereas neutrino is a massless neutral particle. So the nonzero magnetic moment can only be obtained through the weak interaction of the neutrino with the corresponding charged lepton as shown in Fig. 1. In the standard model massless and neutral neutrino cannot interact with the magnetic field and exhibits zero magnetic moment. It is a higher order process if neutrino has some mass. In this way, the magnetic moment of the neutrino will depend on the extension of the standard model and will be a model dependent quantity. We just consider the minimally extended standard model with the neutrino mass as $1 \mathrm{MeV}$ just to compare the thermal mass of the electron and the magnetic

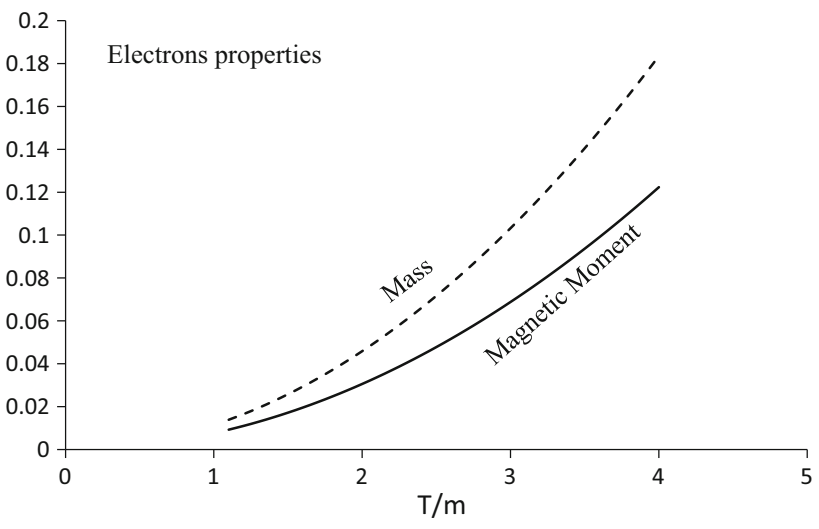

Fig. 3 A comparison of temperature dependent self-mass and the corresponding magnetic moment of the electron shows that the behavior of magnetic dipole moment at finite temperature is exactly similar to the mass of the electron at high temperatures, i.e; $T>m$

dipole moment of the neutrino as a function of temperature. This comparison is done at extremely high temperatures. The induced magnetic moment of the neutrinos is plotted in Fig. 4, where the magnetic moment of the neutrino is plotted as a function of temperature for the upper limit of the mass of the electron type neutrino around $1 \mathrm{eV}$. In this first order correction, the minimal standard model obeying the conservation of the individual lepton number is considered.

Temperature correction is suppressed for neutrino because the temperatures is compared to the mass of $\mathrm{W}$, instead of the electron as W boson is the loop partner of the electron in the bubble diagram. Therefore, the background contribution to 


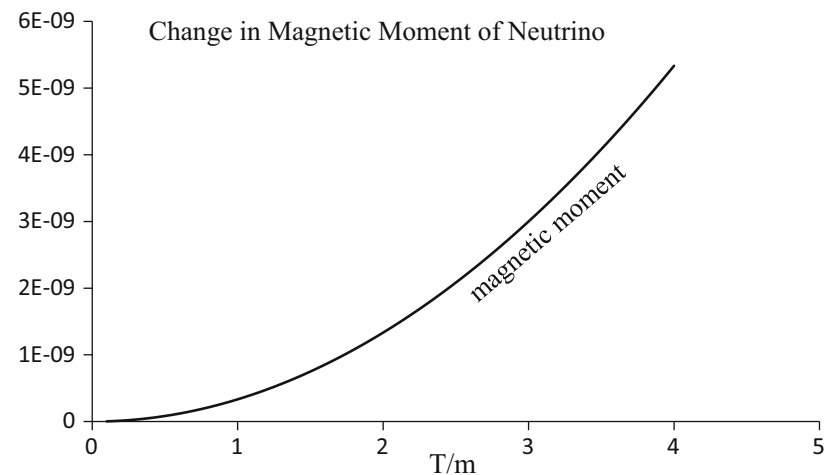

Fig. 4 The ratio of (thermal) electrons background corrections to the magnetic moment of the electronic type neutrino with the corresponding vacuum value of the magnetic moment is plotted as a function of temperature below the neutrino decoupling temperature to extract the pure background effect

the magnetic moment of neutrino is induced as almost $10^{-10}$ times the corresponding contributions to the electron mass which is exactly of the order of $\left(\mathrm{m}^{2} / \mathrm{M}^{2}\right)$ and is of the order of the square of the ratios between the mass of the electron and the mass of $\mathrm{W}$ boson. Figure 4 shows that this induced magnetic moment of the neutrino in the minimal standard model is negligibly small, being a higher order effect.

An explicit comparison of all these values is given in Table 1. It shows the values of all of the QED parameters such as electron properties and the magnetic moment of the electron for a given value of temperature including the induced magnetic moment of the neutrino. However, it is clear from the last column of the table that the ratio of the magnetic moment of the electron type neutrino in a lepton number conserving minimally extended standard model with that of the electron is constant which is actually proportional to the ratio between their masses.

The magnetic moment is a property of the mass. The thermal corrections to the magnetic dipole moment of the neutrino are actually due to its swallowed mass in thermal background. This expected behavior is demonstrated in a plot of the electron mass and its corresponding modification in the dipole moment as a function of temperature. Figure 3 shows a clear demonstration of this behavior of the electron.

Table 1 Electron self-mass, charge, magnetic moment and neutrino magnetic moment have been evaluated for the same values of temperatures in the units of the electron mass and below the coupling tem-
It is also interesting to note the vacuum polarization contribution due to the presence of fermions in the background for $T>m$. Neutrino magnetic moment can be induced the same way even if the neutrinos are not decoupled (below $2 \mathrm{MeV}$ ) as they acquire the induced magnetic dipole moment by the electrons which can occur even if the neutrino concentration is lower but there are enough electrons in the medium.

The properties of the photons at high temperature Photon, as a quanta of energy acquires nonzero mass in a medium with an abundance of electrons with extremely high energy at high temperature. The electromagnetic interaction of the photon with electrons in a medium gives a dynamically generated mass to the photon which can be treated as the screening mass and the Debye shielding turns the medium into an electron-photon relativistic plasma under suitable conditions. The photon is massless at lower temperature and density and the phase transition in a medium occurs at temperatures greater than $1 \mathrm{MeV}$ where the fermion background starts to contribute to the self-energy of the photon which leads to Debye shielding due to the dynamically generated mass of the photons. The behavior of the dynamically generated screening mass and Debye shielding length as a function of temperature is given in Fig. 5. The Debye screening length decreases with the increase in temperature and with the increase in screening mass of the photon. At a temperature around 3-4 MeV, the Debye length decreases, compared

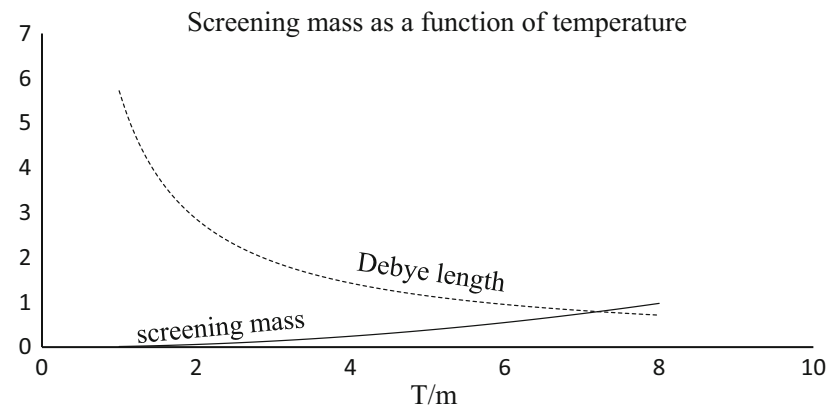

Fig. 5 Plot of plasma screening mass (solid line) and the corresponding Debye shielding length (broken line) as a function of temperature in units of the electron mass

peratures. The chemical potential is ignored at this point to keep this comparison simple

\begin{tabular}{llllll}
\hline$T / m$ & $\delta m / m$ & $\Delta e / e$ & Electron dipole moment & Neutrino dipole moment & Ratio of magnetic moments \\
\hline 1.1 & 0.013879 & $2.34 \mathrm{E}-07$ & 0.009252 & $4.03333 \mathrm{E}-10$ & $4.36 \mathrm{E}-08$ \\
1.5 & 0.025808 & $8.1 \mathrm{E}-07$ & 0.017205 & $7.5 \mathrm{E}-10$ & $4.36 \mathrm{E}-08$ \\
2 & 0.04588 & $2.56 \mathrm{E}-06$ & 0.030587 & $1.33333 \mathrm{E}-09$ & $4.36 \mathrm{E}-08$ \\
2.5 & 0.071688 & $6.25 \mathrm{E}-06$ & 0.047792 & $2.08333 \mathrm{E}-09$ & $4.36 \mathrm{E}-08$ \\
3 & 0.10323 & $1.3 \mathrm{E}-05$ & 0.06882 & 0.000000003 & $4.36 \mathrm{E}-08$ \\
\hline
\end{tabular}


Fig. 6 Plot of propagation velocity and the refractive index in an extremely hot medium

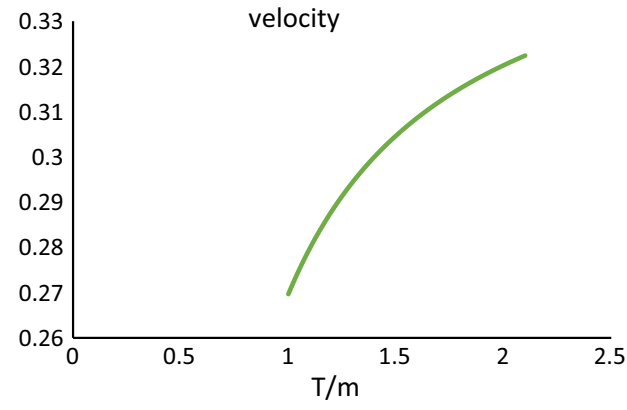

Refractive Index

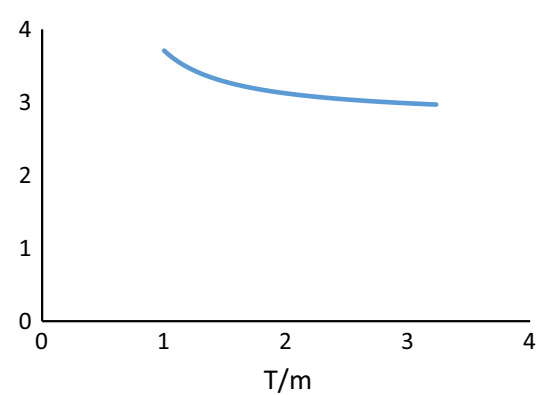

to the screening mass itself. The Debye length corresponds to the potential energy and decrease in the potential energy is expected with temperature.

The number of particles in the Debye sphere is calculated from the Debye shielding length $\lambda_{\mathrm{D}}$, if the number density remains unchanged. We have

$N_{\mathrm{D}}=4 / 3 \pi n_{0} \lambda_{\mathrm{D}}^{3}$

Thus the decrease in $\lambda_{\mathrm{D}}$ with temperature is associated with the number density of the universe. The composition of the universe changes with temperature and so does the $N_{\mathrm{D}}$ due to the change in mass as well as the other parameters of the theory such as $\lambda_{\mathrm{D}}$ and the propagation velocities.

We consider the small longitudinal component and the magnitude of the wave-vector $k$ is much larger than the frequency $\omega$, making it a very high energy wave.

However, the velocity of waves in a medium grows quickly with temperature whereas the refractive index decreases relatively slowly with temperature in such a medium (Fig. 6).

The above results indicate that QED can be used as the only theory below the neutrino decoupling temperature and that is $2 \mathrm{MeV}$. As soon as the temperature of the system crosses this limit, concentration of the neutrinos become significant and the weak interactions come into play, some of the energy is used in weak interaction and is not ignorable with the rise of temperature.

It can be clearly seen that the thermal corrections lead to a quadratic increase in the measurable values of the physical parameters of the theory (Figs. 2, 3, and 4). However, the interaction-based bulk properties such as the propagation speed, magnetic moment and the screening mass show different behavior. This difference in behavior balances some of the effects and keeps open the possibility of the existence of physical systems at extreme temperatures (kinetic energies) and large densities or chemical potential (potential energies) and magnetic field helping to develop an equilibrium and maintain the system.

The properties of the neutrinos are related to the massive neutrinos, which opens up a whole list of possible extensions of the standard model to accommodate massive neutrinos. The thermal effects on the properties of the neutrinos are highly model dependent [21-24] and can only be calculated individually for every model. Even the Dirac and Majorana mass will contribute differently to the magnetic moment.

It can be clearly seen that all the above equations of Sect. 4 reproduce the already existing results in the tranversality limit where $\omega^{2} \approx k^{2}$, we see that propagation velocity, electric permittivity and magnetic permeability are all equal to unity.

Open Access This article is distributed under the terms of the Creative Commons Attribution 4.0 International License (http://creativecomm ons.org/licenses/by/4.0/), which permits unrestricted use, distribution, and reproduction in any medium, provided you give appropriate credit to the original author(s) and the source, provide a link to the Creative Commons license, and indicate if changes were made.

Funded by SCOAP S $^{3}$

\section{References}

1. P. Landsman, Ch.G Weert, Phys. Rep. 145, 141 (1987), and references therein

2. L. Dolan, R. Jackiw, Phys. Rev. D 9, 3320 (1974)

3. A. Weldon, Phys. Rev. D 26, 1394 (1992)

4. R. Kobes, G.W. Semenoff, Nucl. Phys. B 260, 714 (1985)

5. R. Kobes, G.W. Semenoff, Nucl. Phys. B 272, 329 (1986)

6. S.S. Masood, Phys. Res. Int. 2014, 48913 (2014). arXiv: 1407.1414

7. K. Ahmed, S.S. Masood, Phys. Rev. D 35, 1861 (1987)

8. K. Ahmed, S.S. Masood, Phys. Rev. D 35, 4020 (1987)

9. K. Ahmed, S.S. Masood, Ann. Phys. 164, 460 (1991)

10. S.S. Masood, Phys. Rev. D 44, 3943 (1991)

11. S.S. Masood, Phys. Rev. D 47, 648 (1993), and references therein

12. M. Haseeb, S.S. Masood, Phys. Lett. B 704, 66 (2011). and references therein

13. S.S. Masood, JMP 5, 296 (2014). arXiv:1405.1239

14. E.J. Levinson, D.H. Boal, Phys. Rev. D 31, 3280 (1985)

15. S.S. Masood, Phys. Rev. D 36, 2602 (1987)

16. S.S. Masood, Phys. Rev. D 48, 3250 (1993), and references therein

17. D. Notzold, G. Raffelt, Nucl. Phys. B 307, 924 (1988)

18. C.S. Lim, W.J. Marciano, Phys. Rev. D 37, 1368 (1988)

19. S.S. Masood, Astropart. Phys. 4, 189 (1995)

20. S. S. Masood, JHEPGC 01(01), 56270 (2015). arXiv:1506.01284 [hep-phys]. https://doi.org/10.4236/jhepgc.2015.11001

21. R. Barbieri, P. Mohapatra, Phys. Lett. B 61, 23 (1988)

22. K.S. Babu, V.S. Mathur, Phys. Lett. B 196, 218 (1987)

23. Pal, Mohapatra, Massive Neutrinos in Physics and Astrophysics (World Scientific Publication, Singapore, 1991)

24. B.C. Canas et al., Phys. Lett. B 753, 191 (2016) 